TITLE:

\title{
Theoretical studies on magnetic circular dichroism by the finite perturbation method with relativistic corrections
}

\section{$\operatorname{AUTHOR}(\mathrm{S}):$}

Honda, Y; Hada, M; Ehara, M; Nakatsuji, H; Michl, J

\section{CITATION:}

Honda, Y ... [et al]. Theoretical studies on magnetic circular dichroism by the finite perturbation method with relativistic corrections. JOURNAL OF CHEMICAL PHYSICS 2005, 123(16): 164113.

ISSUE DATE:

2005-10-22

URL:

http://hdl.handle.net/2433/50104

\section{RIGHT:}

Copyright 2005 American Institute of Physics. This article may be downloaded for personal use only. Any other use requires prior permission of the author and the American Institute of Physics. 


\title{
Theoretical studies on magnetic circular dichroism by the finite perturbation method with relativistic corrections
}

\author{
Y. Honda ${ }^{\text {a),b) }}$ and M. Hada \\ Department of Chemistry, Graduate School of Science, Tokyo Metropolitan University, \\ 1-1 Minami-Osawa, Hachioji-shi, Tokyo 192-0397, Japan \\ M. Ehara and H. Nakatsuji ${ }^{\text {a) }}$ \\ Department of Synthetic Chemistry and Biological Chemistry, Graduate School of Engineering, \\ Kyoto University, Nishikyo-ku, Kyoto 615-8510, Japan \\ J. Michl \\ Department of Chemistry and Biochemistry, University of Colorado, Boulder, Colorado, 80309-0215
}

(Received 29 June 2005; accepted 29 August 2005; published online 27 October 2005)

\begin{abstract}
A theoretical method for calculating magnetic circular dichroism (MCD) of molecules is presented. We examined the numerical accuracy and the stability of the finite perturbation (FP) method and the sum-over-state (SOS) perturbation method. The relativistic effects are shown to be important for the MCD spectra of molecules containing heavy elements. Calculations using the FP and the SOS methods were carried out for ethylene, para- and ortho-benzoquinone, showing that the FP method is superior to the SOS method, as expected. The relativistic effect was examined using the second-order Douglas-Kroll Hamiltonians for the halogen molecules $\mathrm{F}_{2}, \mathrm{Cl}_{2}, \mathrm{Br}_{2}$, and $\mathrm{I}_{2}$. The Faraday terms of $\mathrm{I}_{2}$ and $\mathrm{Br}_{2}$ were strongly affected by the relativistic effects, while the effect was negligible for $\mathrm{Cl}_{2}$ and $\mathrm{F}_{2}$. () 2005 American Institute of Physics. [DOI: 10.1063/1.2080027]
\end{abstract}

\section{INTRODUCTION}

Magnetic circular dichroism (MCD) is the differential absorption of dextrorotatory and levorotatory circular polarized light in the presence of an external magnetic field. The MCD spectrum is often used as a complement to the corresponding electronic absorption spectrum, since the former has a set of positive and negative peaks while the latter always has positive ones. MCD is therefore particularly useful in assigning spectral bands containing multiple overlapping electronic and vibrational peaks. MCD has been observed for simple polyatomic molecules, e.g., acetylene, ${ }^{1}$ ethylene, ${ }^{2,3}$ tetramethylethylene, ${ }^{4} \quad$ cyclopropane, ${ }^{5}$ benzene derivatives, ${ }^{6-12}$ quinones, ${ }^{13}$ etc., and recently for more complex systems such as porphyrins, ${ }^{14}$ porphycenes,${ }^{15}$ metal complexes, ${ }^{16}$ and so on.

The basic theory of MCD was firmly established by the $1970 \mathrm{~s},{ }^{17}$ and many theoretical studies on this subject have been published. ${ }^{9,13,18-25}$ Shieh et al. reported a theory of molecules in dense media, ${ }^{18}$ and Coriani et al. reported a formulation in the framework of the coupled-cluster response theory. ${ }^{19}$ A number of calculations using $a b$ initio $^{20,21}$ and semiempirical molecular-orbital theories have been performed on ethylene ${ }^{20}$ cyclopropane ${ }^{21}$ formaldehyde,${ }^{22}$ benzene derivatives, ${ }^{9,23,24}$ quinones, ${ }^{13,20}$ and aromatic $^{24}$ and nonaromatic ${ }^{25}$ cyclic $\pi$-electron compounds. The MCD is characterized by the Faraday terms which consist of the $A, B$, and $C$ terms. The $A$ and $C$ terms can readily be calculated, whereas the calculations of the $B$ term are somewhat com-

\footnotetext{
a) Authors to whom correspondence should be addressed.

b) Electronic mail: honda@comp.metro-u.ac.jp
}

plicated, because the formers do not need the magnetic-field derivatives of wave functions while the latter needs them. The details of the Faraday terms will be explained in Sec. II.

We have two purposes in the present paper. One is a comparison of the finite perturbation (FP) method ${ }^{23}$ with the sum-over-states (SOS) method in the calculations of the Faraday $B$ term. The SOS method has commonly been used for the calculations of the $B$ term in the previous studies. $9,13,18,21,22,24,25$ This method has a merit that one does not need to calculate wave functions in magnetic fields. However, the SOS method has some disadvantages: (1) it is time consuming because it involves the calculations of the intermediate states, (2) a convergence problem arises owing to a truncation on the sum over the intermediate states, (3) the usual SOS method does not provide the best results except with full configuration interaction (CI), and some inaccuracy remains even if all intermediate states are considered. This point was discussed for the second-order SOS perturbation energy based on the Hartree-Fock wave function in Ref. 26. It is briefly revisited in the next section. On the other hand, the FP method and quadratic response theory ${ }^{20}$ are free from these disadvantages. (The latter has been employed by Coriani et al. ${ }^{19,20}$ ) In this paper, we calculate MCD by the FP and the SOS methods and show the effects of (2) and (3) in terms of practical computational results. This comparison is performed for ethylene, $p$-benzoquinone (BQ), and $o$-BQ, and these are discussed in Sec. IV.

The other purpose of this paper is to examine relativistic effects on the MCD spectra, and these are discussed in Sec. $\mathrm{V}$. The relativistic effect is expected to be important for the MCD of molecules containing heavy elements, e.g., halogen molecules, ${ }^{27}$ methyl halides, ${ }^{28,29}$ and carbon tetrahalides. ${ }^{30}$ 
Recently, we have developed the quasirelativistic generalized unrestricted Hartree-Fock ${ }^{31-33}$ (GUHF) single excitation configuration interaction (SECI) method and have reported that the relativistic correction is essential in the calculation of the Faraday term for methyl iodide. ${ }^{34}$ In this paper, the relativistic effects in the halogen molecules $\mathrm{I}_{2}, \mathrm{Br}_{2}, \mathrm{Cl}_{2}$, and $\mathrm{F}_{2}$ are investigated by the GUHF/SECI method.

\section{FORMULATION OF MCD AND FARADAY TERMS}

\section{A. MCD ellipticity}

The working equations used in the present study are derived from the basic formula of MCD given in Ref. 17. These equations are used in the framework of both relativistic and nonrelativistic theories. Relativistic effects are introduced as a natural consequence of the wave functions determined by the relativistic zeroth-order Hamiltonians and the relativistic form of the magnetic field.

When an electromagnetic field of light traveling in the $z$ direction is applied to a molecule, the ellipticity $\theta$ caused by the electronic transition from the ground state $a$ to the excited state $j$ within the long-wavelength approximation is given by

$$
\theta=-N_{a} \frac{4 \pi^{3}}{h c} f\left(\omega_{j a}, \omega\right) \operatorname{Im}\left(m_{x}^{*} m_{y}\right),
$$

where $N_{a}$ is the number of ground-state molecules in the light path, $f\left(\omega_{j a}, \omega\right)$ denotes a spectral shape function of the energy $\omega$, and $\omega_{j a}$ is the excitation energy for $a \rightarrow j$. The terms $m_{x}$ and $m_{y}$ are the electric transition dipole moments. In the absence of an external magnetic field, $\theta$ in Eq. (1) is zero, but it is nonzero in the presence of a magnetic field. [Circular dichroism (CD) of optically active molecules is not derived from Eq. (1), because the long-wavelength approximation is inappropriate for the description of $\mathrm{CD}$.] The MCD spectrum is obtained by differentiating $\theta$ with respect to the external magnetic-field strength $H_{z}$ in the limit of $H_{z} \rightarrow 0$. The MCD ellipticity is given by

$$
\begin{aligned}
\left(\frac{\partial \theta}{\partial H_{z}}\right)_{H_{z}=0}= & -\frac{4 \pi^{3}}{h c}\left[N_{a}\left(\frac{\partial f\left(\omega_{j a}, \omega\right)}{\partial H_{z}}\right)_{0} \operatorname{Im}\left(m_{x}^{*} m_{y}\right)\right. \\
& +N_{a} f\left(\omega_{j a}, \omega\right)\left(\frac{\partial \operatorname{Im}\left(m_{x}^{*} m_{y}\right)}{\partial H_{z}}\right)_{0} \\
& \left.+\left(\frac{\partial N_{a}}{\partial H_{z}}\right)_{0} f\left(\omega_{j a}, \omega\right) \operatorname{Im}\left(m_{x}^{*} m_{y}\right)\right]
\end{aligned}
$$

The derivative in the first term is rewritten as

$$
\left(\frac{\partial f\left(\omega_{j a}, \omega\right)}{\partial H_{z}}\right)_{0}=\sum_{j \leftarrow a}\left(\frac{\partial f\left(\omega_{j a}, \omega\right)}{\partial \omega_{j a}}\right)_{0}\left(\frac{\partial \omega_{j a}}{\partial H_{z}}\right)_{0},
$$

where the summation over $j \leftarrow a$ means the sum of all pairs of degenerate substates $a$ and $j$. When the magnetic dipole moments are zero both for $a$ and $j$, namely, neither $a$ nor $j$ is degenerate, then $\omega_{j a}$ is unaffected by a magnetic field, and this term becomes zero. However, if $a$ or $j$ is degenerate, the degeneracy vanishes in the presence of the magnetic field, and the energies of the states are shifted by

$$
-\mu_{x x}^{z} H_{z} \quad(x=a, j),
$$

where

$$
\mu_{a a}^{z}=\left\langle a\left|\hat{\mu}_{z}\right| a\right\rangle, \quad \mu_{j j}^{z}=\left\langle j\left|\hat{\mu}_{z}\right| j\right\rangle .
$$

In Eq. (5), $\mu_{z}$ is the $z$ component of the magnetic moment and is given by

$$
\hat{\mu}_{z}=\frac{e}{2 m_{e} c}\left((\hat{\boldsymbol{r}} \times \hat{\boldsymbol{p}})_{z}+g \hat{S}_{z}\right),
$$

where $g$ is the $g$ factor of a free electron. Substituting Eq. (4) into Eq. (3), we obtain

$$
\left(\frac{\partial f\left(\omega_{j a}, \omega\right)}{\partial H_{z}}\right)_{0}=\left(-\frac{1}{\hbar}\right) \sum_{j \leftarrow a}\left(\frac{\partial f\left(\omega_{j a}, \omega\right)}{\partial \omega_{j a}}\right)_{0}\left(\mu_{j j}^{z}-\mu_{a a}^{z}\right) .
$$

On the other hand, the third term of Eq. (2) is nonzero only when the ground state $a$ is degenerate. $N_{a}$ is assumed to be determined by the Boltzmann distribution since the molecules are populated among the split substates in the presence of a magnetic field. The number of molecules in the $\alpha$ th substate of the ground state $a, N_{a}^{\alpha}$, is given by

$$
\begin{aligned}
N_{a}^{\alpha} & =N_{a} \frac{\exp \left(-E_{a}^{\alpha} / k T\right)}{\sum_{\alpha^{\prime}}^{d_{a}} \exp \left(-E_{a}^{\alpha^{\prime}} / k T\right)} \\
& =N_{a}\left[\sum_{\alpha^{\prime}}^{d_{a}} \exp \left(\frac{E_{a}^{\alpha}-E_{a}^{\alpha^{\prime}}}{k T}\right)\right]^{-1},
\end{aligned}
$$

where $d_{a}$ is the number of the degenerate substates of $a, k$ is the Boltzmann constant, and $T$ is the absolute temperature. Since the magnitude of the energy splitting is much smaller than the thermal energy $k T$,

$$
\begin{aligned}
N_{a}^{\alpha} & \approx N_{a}\left[\sum_{\alpha^{\prime}}^{d_{a}}\left(1+\frac{E_{a}^{\alpha}-E_{a}^{\alpha^{\prime}}}{k T}\right)\right]^{-1}=\frac{N_{a}}{d_{a}}\left(1+\frac{E_{a}^{\alpha}-E_{a}^{0}}{k T}\right)^{-1} \\
& \approx \frac{N_{a}}{d_{a}}\left(1+\frac{E_{a}^{0}-E_{a}^{\alpha}}{k T}\right) .
\end{aligned}
$$

Therefore, Eq. (2) can be written as

$$
\begin{aligned}
\left(\frac{\partial \theta}{\partial H_{z}}\right)_{H_{z}=0}= & -\frac{N_{a}}{d_{a}} \frac{4 \pi^{3}}{h c} \sum_{j \leftarrow a}\left[\left(-\frac{1}{\hbar}\right)\left(\frac{\partial f\left(\omega_{j a}, \omega\right)}{\partial \omega_{j a}}\right)_{0}\left(\mu_{j j}^{z}-\mu_{a a}^{z}\right)\right. \\
& \times \operatorname{Im}\left(m_{x}^{*} m_{y}\right)+f\left(\omega_{j a}, \omega\right)\left(\frac{\partial \operatorname{Im}\left(m_{x}^{*}, m_{y}\right)}{\partial H_{z}}\right)_{0} \\
& \left.+\left(\frac{\mu_{a a}^{z}}{k T}\right) f\left(\omega_{j a}, \omega\right) \operatorname{Im}\left(m_{x}^{*} m_{y}\right)\right] .
\end{aligned}
$$

\section{B. Faraday terms}

The MCD ellipticity is characterized by three parameters $A, B$, and $C$, which are called the Faraday terms, and one shape function $f\left(\omega_{j a}, \omega\right)$, as follows: 


$$
\begin{aligned}
\left(\frac{\partial \theta}{\partial H_{z}}\right)_{H_{z}=0}= & -N_{a} \frac{4 \pi^{2}}{3 h c}\left[\left(-\frac{1}{\hbar}\right)\left(\frac{\partial f\left(\omega_{j a^{\prime}} \omega\right)}{\partial \omega_{j a}}\right)_{0} A_{z}\right. \\
& \left.+f\left(\omega_{j a}, \omega\right)\left(B_{z}+\frac{C_{z}}{k T}\right)\right] .
\end{aligned}
$$

Comparing Eq. (10) with Eq. (11), we define the following three terms:

$$
\begin{aligned}
& A_{z}=\frac{3}{d_{a j \leftarrow a}} \sum_{j}\left(\mu_{j j}^{z}-\mu_{a a}^{z}\right) \operatorname{Im}\left(m_{x}^{*} m_{y}\right), \\
& B_{z}=\frac{3}{d_{a}} \sum_{j \leftarrow a}\left(\frac{\partial \operatorname{Im}\left(m_{x}^{*} m_{y}\right)}{\partial H_{z}}\right)_{0}, \\
& C_{z}=\frac{3}{d_{a j \leftarrow a}} \sum_{a a}^{z} \operatorname{Im}\left(m_{x}^{*} m_{y}\right) .
\end{aligned}
$$

Since the Faraday terms are determined only by the electric and magnetic moments of the molecule, these values are intrinsic to the electronic states of the molecule. The parameters $A, B$, and $C$ can be determined experimentally.

In gas and liquid phases, one has to calculate the isotropic average of Eq. (12). They are given by

$$
\begin{aligned}
A & =\frac{A_{x}+A_{y}+A_{z}}{3}=\frac{1}{2 d_{a}} \sum_{\alpha \beta \gamma} \varepsilon_{\alpha \beta \gamma} \sum_{j \leftarrow a}\left(\mu_{j j}^{z}-\mu_{a a}^{z}\right) \operatorname{Im}\left(m_{x}^{*} m_{y}\right), \\
B & =\frac{B_{x}+B_{y}+B_{z}}{3} \\
& =\frac{1}{2 d_{a}} \sum_{\alpha \beta \gamma} \varepsilon_{\alpha \beta \gamma} \sum_{j \leftarrow a}\left[\frac{\partial}{\partial H_{\gamma}} \operatorname{Im}\left(m_{a j, \alpha}^{(\gamma)} m_{j a, \beta}^{(\gamma)}\right)\right]_{H_{\gamma}=0}, \\
C & =\frac{C_{x}+C_{y}+C_{z}}{3}=\frac{1}{2 d_{a}} \sum_{\alpha \beta \gamma} \varepsilon_{\alpha \beta \gamma} \sum_{j \leftarrow a} \mu_{a a}^{\gamma} \operatorname{Im}\left(m_{a j, \alpha} m_{j a, \beta}\right),
\end{aligned}
$$

where $\varepsilon$ is Levi-Civita tensor. The indices $\alpha, \beta$, and $\gamma$ run over $x, y$, and $z$. The terms $m_{j a, \alpha}$ and $m_{j a, \alpha}^{(\gamma)}$ mean the $\alpha$-direction components of the electric transition moments between $a$ and $j$ without a magnetic field and that in a $\gamma$-direction magnetic field $H_{\gamma}$, respectively.

The $A$ and $C$ terms consist of the magnetic moments of the unperturbed ground and excited states and the electric transition moments, while the $B$ term is the first-order derivatives of the magnetic field. In the FP method, the $B$ term is obtained by numerical differentiation of Eq. (13) using the wave functions in a finite magnetic field, namely,

$$
B=\frac{1}{2 d_{a}} \sum_{\alpha \beta \gamma} \varepsilon_{\alpha \beta \gamma} \lim _{H_{\gamma} \rightarrow 0} \sum_{j \leftarrow a} \operatorname{Im} \frac{m_{a j, \alpha}^{(\gamma)} m_{j a, \beta}^{(\gamma)}-m_{a j, \alpha} m_{j a, \beta}}{H_{\gamma}},
$$

where

$$
m_{a j, \alpha}^{(\gamma)}=\left\langle\Psi_{a}\left(H_{\gamma}\right)\left|\hat{m}_{\alpha}\right| \Psi_{j}\left(H_{\gamma}\right)\right\rangle,
$$

where $\Psi_{a}\left(H_{\gamma}\right)$ and $\Psi_{j}\left(H_{\gamma}\right)$ are the perturbed wave functions in the presence of $H_{\gamma}$. Alternatively, using the first-order perturbed $a$ and $j$ states,

$$
\begin{aligned}
& \left|a^{(\gamma)}\right\rangle=|a\rangle-H_{\gamma} \sum_{n \neq a} \frac{\left\langle n\left|\hat{\mu}_{\gamma}\right| a\right\rangle}{E_{a}-E_{n}}|n\rangle, \\
& \left|j^{(\gamma)}\right\rangle=|j\rangle-H_{\gamma} \sum_{n \neq j} \frac{\left\langle n\left|\hat{\mu}_{\gamma}\right| j\right\rangle}{E_{j}-E_{n}}|n\rangle,
\end{aligned}
$$

the isotropic $B$ term in the SOS form is given by

$$
\begin{aligned}
B= & \frac{1}{d_{a j \leftarrow a}} \sum_{\operatorname{Im}}\left[\sum_{n \neq a} \frac{\boldsymbol{\mu}_{n a} \cdot\left(\boldsymbol{m}_{a j} \times \boldsymbol{m}_{j n}\right)}{E_{n}-E_{a}}\right. \\
& \left.+\sum_{n \neq j} \frac{\boldsymbol{\mu}_{j n} \cdot\left(\boldsymbol{m}_{a j} \times \boldsymbol{m}_{n a}\right)}{E_{n}-E_{j}}\right] .
\end{aligned}
$$

If $n$ in Eq. (17) runs over a complete set of eigenstates, Eqs. (14) and (17) give the same results. However, practically, the summation over $n$ is usually truncated. Furthermore, even if we employ the complete HF/SECI approximation for the ground and excited states, the Faraday $B$ term is somewhat troublesome, because the intermediate state $n$ in Eq. (17) runs over the SECI wave functions and the SECI state $j$ has interactions with single and double excitation CI (SDCI) through the perturbation with respect to the magnetic field. It therefore turns out that even if all the SECI states are considered as the intermediate states $n$, the best value of the $B$ term within the SECI approximation will not be obtained. This error is not specific to the case of the SECI approximation, but always arises except for the full CI, and it can be substantial in some cases.

\section{COMPUTATIONAL DETAILS}

\section{A. Molecules}

Calculations of the Faraday terms of ethylene, $p$-BQ, and $o$ - $\mathrm{BQ}\left(\mathrm{C}_{6} \mathrm{H}_{4} \mathrm{O}_{2}\right)$ were carried out using a nonrelativistic method, in order to compare the FP and SOS methods. Subsequently, calculations of halogen molecules $\left(\mathrm{I}_{2}, \mathrm{Br}_{2}, \mathrm{Cl}_{2}\right.$, and $\mathrm{F}_{2}$ ) were carried out using both relativistic and nonrelativistic methods. The geometrical parameters of these molecules were taken from Refs. 35-38 for ethylene, $p$-BQ, $o$-BQ, and halogens, respectively.

\section{B. Basis set}

The Huzinaga-Dunning $(9 s 5 p / 4 s) /[4 s 2 p / 2 s]$ (DZ) basis sets with standard scaling factors ${ }^{39}$ were used for $\mathrm{C}, \mathrm{O}$, and $\mathrm{H}$ atoms in the calculations on ethylene, $p$-BQ, and $o-\mathrm{BQ}$. The reason we employed these small basis sets is that the nontruncated SOS (sum over "all" the intermediate states) calculations are needed to study the difference between the results of the SOS and FP methods, rather than comparing the results to the experimental values. For $p$-BQ and $o-\mathrm{BQ}$, however, the calculations with $[4 s 2 p 1 d / 2 s]$ (DZP) sets were also performed. In the calculations on halogens, we used the Huzinaga-Dunning $(9 s 5 p) /[5 s 3 p]$ plus the Huzinaga $[2 d]$ polarization $^{40}$ and the Jungen $[5 s 5 p]$ Rydberg functions ${ }^{41}$ for F; the McLean-Chandler (13s10p)/[6s5p] (Ref. 42) plus the Huzinaga $[2 d]$ polarization and the Jungen $[5 s 4 p]$ Rydberg functions for $\mathrm{Cl}$; the Huzinaga well-tempered Gaussian (WTG) $(26 s 20 p 14 d) /[8 s 8 p 5 d]$ (Ref. 43) plus the Jungen 
[5s5p] Rydberg functions for $\mathrm{Br}$; and the Huzinaga WTG $(28 s 23 p 17 d) /[11 s 8 p 6 d]$ plus the Huzinaga $[1 d]$ polarization and the Jungen $[5 s 5 p]$ Rydberg functions for I. The WTG basis sets for $\mathrm{Br}$ and I were contracted using second-order Douglas-Kroll calculations.

\section{Hamiltonians}

The levels of nonrelativistic and relativistic Hamiltonians examined here are abbreviated as follows:

(a) Non-Rel: nonrelativistic Hamiltonian,

(b) SFR: spin-free (scalar) relativistic,

(c) SFR+SO1:SFR+one-electron spin-orbit interaction, and

(d) Full-Rel $(\mathrm{SFR}+\mathrm{SO} 1+\mathrm{SO} 2): \mathrm{SFR}+\mathrm{SO} 1+$ two-electron spin-orbit interaction.

The SFR and SO1 terms were generated by the second-order Douglas-Kroll-Hess method. ${ }^{44-46}$ The $\mathrm{SO} 2$ term is introduced by the Breit-Pauli-type two-electron spin-orbit interaction. For the perturbation term due to the magnetic field, the nonrelativistic form was used in the calculations. In the previous study, we investigated the effect of the DouglasKroll transformation of the magnetic dipole operator in the calculations of NMR chemical shifts. ${ }^{32}$ In that study, we found that this effect is small unless the electrons in the vicinity of heavy nuclei are treated. In the present case, we are interested in the outer-valence excitations, and therefore we believe that the nonrelativistic treatment is enough for the magnetic dipole operator.

\section{Wave functions}

The ground/excited-state wave functions were calculated by the GUHF/SECI method. ${ }^{31-34}$ The inner-core orbitals of atoms other than $\mathrm{H}$ were frozen in the SECI calculations. Although the SECI approximation gives rather poor excitation energies compared to electron correlation theories, e.g., multireference (MR)-SDCI, complete active space secondorder perturbation (CASPT2), ${ }^{47-49}$ and the symmetryadapted cluster-CI (SAC-CI) method, ${ }^{50-52}$ the MCD Faraday terms are expected to be qualitatively valid, because they essentially reflect one-electron processes. For halogens, however, the SAC-CI calculations with the SO1 term were also carried out in order to evaluate the electron correlation effect.
The SAC-CI wave functions with the SO1 term were obtained by the diagonalization of the SO1 Hamiltonian matrix represented by the unperturbed singlet and triplet SAC-CI states. $^{34,53}$

\section{E. Methods of calculating the $B$ terms}

The SOS and FP methods were employed in the calculation of the $B$ terms of ethylene, $p-\mathrm{BQ}$, and $o-\mathrm{BQ}$, and the results of these two methods were compared. For halogen molecules, only the FP method was used, because the purpose was not a comparison of the SOS and FP methods, but the examination of the relativistic effects. The gauge origin was taken to be at the center of gravity of the molecule. The gauge dependence of the Faraday terms was also examined, and we confirmed that the dependence was negligible for the molecules studied here.

\section{COMPARISON BETWEEN THE SOS AND FP METHODS}

In this section, we study the difference between the results calculated by the SOS and FP methods for ethylene, $p$-BQ, and $o-\mathrm{BQ}$, rather than comparing the results to the experimental values. Table I shows the SECI excitation energies and the MCD Faraday $B$ terms for some singlet excited states of ethylene using the SOS and FP methods. Since all the states are nondegenerate, the Faraday $A$ and $C$ terms disappear. The Faraday $B$ terms calculated by the FP method are nearly constant with respect to the strength of the finite magnetic field. However, since this system is small, we considered all the SECI states (the number of states $n$ is 108) as intermediate states in the SOS method. It is evident from Table I that there are significant differences in the values of the $B$ terms calculated by the two methods. In particular, the two methods even predict opposite signs for the $1{ }^{1} B_{1 u}$ state (the lowest $\pi-\pi^{*}$ state): the $B$ term calculated by the FP method is about $0.037 \mathrm{D}^{2} \mu \mathrm{B} / \mathrm{kK}$, whereas the SOS method yields -0.0242 . Although the experimental value of the $B$ term for this state is unknown, it is reported to be positive for ethylene $^{2}$ and tetramethylethylene. ${ }^{4}$ Recently, reexamination of the absorption and MCD spectra of ethylene was performed by Snyder et al., and they reported that the $1{ }^{1} B_{1 u}\left(\pi-\pi^{*}\right)$ and the $1{ }^{1} B_{3 u}[\pi$-Ryd $(3 s)]$ states have the opposite MCD signs. ${ }^{54}$ Our results support this finding, as well

TABLE I. SECI excitation energies and Faraday $B$ terms for ethylene calculated by GUHF-SECI with the sum-over-states (SOS) method and finite perturbation (FP) method. The $z$ axis is parallel to the $\mathrm{C}=\mathrm{C}$ bond, and the $x$ axis is perpendicular to the molecular plane.

\begin{tabular}{|c|c|c|c|c|c|}
\hline & \multirow{2}{*}{$\begin{array}{l}\text { SECI } \\
\text { energy } \\
\left(\mathrm{cm}^{-1}\right)\end{array}$} & \multirow[b]{2}{*}{ Nature } & \multirow{2}{*}{$\begin{array}{l}\text { SOS method }^{\mathrm{a}, \mathrm{b}} \\
n=108 \text { (full) }\end{array}$} & \multicolumn{2}{|c|}{ FP method ${ }^{\mathrm{a}, \mathrm{c}}$} \\
\hline & & & & $H=10^{-4}$ a.u. & $H=10^{-5}$ a.u. \\
\hline $1{ }^{1} B_{1 u}$ & 67634 & $\pi-\pi^{*}$ & -0.0242 & 0.0373 & 0.0377 \\
\hline $1{ }^{1} B_{3 u}$ & 89099 & $\pi-\sigma^{*}$ & -0.0005 & -0.0014 & -0.0014 \\
\hline $1{ }^{1} B_{2 u}$ & 113220 & $\sigma-\sigma^{*}$ & -0.1860 & -0.0500 & -0.0503 \\
\hline $2{ }^{1} B_{3 u}$ & 113984 & $\sigma-\sigma^{*}$ & 0.2199 & 0.1376 & 0.1377 \\
\hline $2{ }^{1} B_{1 u}$ & 130120 & $\sigma-\sigma^{*}$ & -0.3202 & -0.4540 & -0.4528 \\
\hline
\end{tabular}


TABLE II. SECI excitation energies and Faraday $B$ terms for $p$-BQ calculated by GUHF-SECI/DZ with the sum-over-states (SOS) method and finite perturbation (FP) method. The $z$ axis is parallel to the $\mathrm{C}=\mathrm{O}$ bonds, and the $x$ axis is perpendicular to the molecular plane.

\begin{tabular}{|c|c|c|c|c|c|c|c|}
\hline & \multirow{2}{*}{$\begin{array}{l}\text { SECI } \\
\text { energy } \\
\left(\mathrm{cm}^{-1}\right)\end{array}$} & \multirow[b]{2}{*}{ Nature } & \multicolumn{3}{|c|}{ SOS method ${ }^{\mathrm{a}, \mathrm{b}}$} & \multirow{2}{*}{$\begin{array}{l}\text { FP method } \\
H=10^{-4} \text { a.u. }\end{array}$} & \multirow{2}{*}{$\begin{array}{c}\text { Expt. }^{\mathrm{a}, \mathrm{c}} \\
\text { In } n \text {-hexane }\end{array}$} \\
\hline & & & $n=29$ & $n=160$ & $n=1040$ & & \\
\hline${ }^{1} B_{1 u}$ & 52159 & $\pi-\pi^{*}$ & -0.2364 & -0.3878 & -0.3931 & $\begin{array}{c}-0.2667 \\
(-0.1791)^{d}\end{array}$ & -0.04 \\
\hline $1{ }^{1} B_{3 u}$ & 67403 & $n-\pi^{*}$ & 0.0020 & 0.0124 & 0.0055 & 0.0795 & \\
\hline $2{ }^{1} B_{3 u}$ & 71719 & $n-\pi^{*}$ & -0.0174 & -0.0223 & -0.0213 & -0.0738 & \\
\hline $2{ }^{1} B_{1 u}$ & 72912 & $\pi-\pi^{*}$ & 1.4002 & 1.5700 & 1.5297 & 1.7213 & \\
\hline
\end{tabular}

as in agreement with the uncorrelated results of Ref. 20. The difference between the SOS and FP results is mainly due to the effects of the doubly excited configurations that are considered in the FP method [see Eq. (38) of Ref. 26].

The calculated results for $p-\mathrm{BQ}$ and $o-\mathrm{BQ}$ are listed in Tables II and III, respectively, where the corresponding experimental values of the $B$ terms are also given. Only the Faraday $B$ terms are nonzero for these molecules. The dimensions of the SECI matrices were 1040, and the eigenstates were obtained by diagonalizing the matrices of dimension 1040. In the truncated SOS method, the $n$ states were chosen in the order of lower SECI energies. For both molecules, the $B$ terms depended considerably on the truncation of the intermediate states in the early stage. For example, the $B$ term for $1{ }^{1} B_{1 u}$ state $\left(\pi-\pi^{*}\right)$ of $p$-BQ varies from $-0.2364 \mathrm{D}^{2} \mu \mathrm{B} / \mathrm{kK}$ for $n=29$ to -0.3878 for $n=160$. On the other hand, the changes from $n=160$ to $n=1040$ (full) were small, indicating that the truncation of the SOS to $n=160$ gives results as accurate as the full SOS calculations of these molecules. However, the differences between the FP and the full SOS results were still sizable. In the worst case, the $1{ }^{1} B_{3 u}$ state of $p$-BQ, the SOS result was less than one-tenth of the FP result. This is also attributed to the effects of the doubly excited configurations in the FP method. ${ }^{26}$

It should be mentioned that although the calculated $B$ terms have the same signs as the experimental results, the agreement of their actual values was rather poor. For the
$1{ }^{1} B_{2}$ state of $o-\mathrm{BQ}$, the $B$ term calculated by the FP method was -0.2471 , while the experimental value was -0.19 , a relatively small difference. However, for the lowest allowed state of $p$-BQ $\left(1{ }^{1} B_{1 u}\right)$, the calculated value was -0.2667 , which is much larger than the experimental value -0.04. Part of this inaccuracy is due to the small basis set used, and the calculated values were somewhat improved by using a larger basis set. The results of using the DZP basis set with the FP method are listed in Tables II and III. For $p$-BQ, the calculated $B$ term was improved from -0.2667 in $\mathrm{DZ}$ to -0.1791 in DZP. The $B$ term for $o$-BQ was also changed from -0.2471 in DZ to -0.2375 in DZP. The other factor involved here is probably the lack of electron correlation, which will have to be included for further improvements in the calculation of the Faraday terms.

\section{RELATIVISTIC EFFECTS IN HALOGEN MOLECULES}

Here, we report the MCD calculations on halogen molecules and discuss the relativistic effects on their MCD spectra. The experimental absorption and MCD spectra of $\mathrm{I}_{2}, \mathrm{Br}_{2}$, and $\mathrm{Cl}_{2}$ were observed by Brith et al. ${ }^{27}$ Though the lowest allowed bands in the absorption spectra for $\mathrm{I}_{2}$ and $\mathrm{Br}_{2}$ are apparently attributed to single electronic excitation, three $n$ $-\sigma^{*}$ excited states are identified using MCD spectra. These are the ${ }^{3} \Pi_{1 u},{ }^{3} \Pi_{0 u}$, and ${ }^{1} \Pi_{u}$ states, in the order of increasing energy. The ${ }^{3} \Pi_{1 u}$ and ${ }^{3} \Pi_{0 u}$ states correspond to triplet states

TABLE III. SECI excitation energies and Faraday $B$ terms for $o$-BQ calculated by GUHF-SECI/DZ with the sum-over-states (SOS) method and finite perturbation (FP) method. The $z$ axis is identical to the $C_{2}$ axis, and the $x$ axis is perpendicular to the molecular plane.

\begin{tabular}{|c|c|c|c|c|c|c|c|}
\hline & \multirow{2}{*}{$\begin{array}{c}\text { SECI } \\
\text { energy } \\
\left(\mathrm{cm}^{-1}\right)\end{array}$} & \multirow[b]{2}{*}{ Nature } & \multicolumn{3}{|c|}{ SOS method ${ }^{\mathrm{a}, \mathrm{b}}$} & \multirow{2}{*}{$\begin{array}{l}\text { FP method }{ }^{\text {a }} \\
H=10^{-4} \text { a.u. }\end{array}$} & \multirow{2}{*}{$\begin{array}{c}\text { Expt. }^{\mathrm{a}, \mathrm{c}} \\
\text { In acetonitrile }\end{array}$} \\
\hline & & & $n=16$ & $n=160$ & $n=1040$ & & \\
\hline $1{ }^{1} B_{1}$ & 24346 & $n-\pi^{*}$ & 0.0023 & 0.0015 & 0.0015 & 0.0025 & small $(+)$ \\
\hline $1{ }^{1} B_{2}$ & 35567 & $\pi-\pi^{*}$ & -0.2459 & -0.2441 & -0.2432 & $\begin{array}{c}-0.2471 \\
(-0.2375)^{\mathrm{d}}\end{array}$ & -0.19 \\
\hline $1{ }^{1} A_{1}$ & 62872 & $\pi-\pi^{*}$ & -0.9427 & -1.0088 & -0.9944 & -0.6679 & \\
\hline $2{ }^{1} B_{1}$ & 62939 & $n-\pi^{*}$ & 0.6854 & 0.6881 & 0.6887 & 0.5149 & very large $(+)$ \\
\hline
\end{tabular}

${ }^{\mathrm{a}}$ Unit in $10^{-3} \mathrm{D}^{2} \mu \mathrm{B} / \mathrm{cm}^{-1}$.

${ }^{\mathrm{b}} n=1040$ is the full intermediate states.

${ }^{c}$ Reference 13.

${ }^{\mathrm{d}}$ Result of DZP basis in parentheses. 
TABLE IV. SECI excitation energies, Faraday $A$ terms $(A)$, Faraday $B$ terms $(B)$, dipole strengths $(D)$, and oscillator strengths $(f)$ of ${ }^{3} \Pi_{1 u},{ }^{3} \Pi_{0 u}$, and ${ }^{1} \Pi_{u}$ states of $\mathrm{I}_{2}$ calculated by GUHF-SECI.

\begin{tabular}{|c|c|c|c|c|c|c|c|c|}
\hline & \multicolumn{5}{|c|}{ GUHF-SECI } & \multirow{2}{*}{$\begin{array}{c}\text { SAC-CI } \\
\text { SO1 }\end{array}$} & \multicolumn{2}{|c|}{ Expt. $^{\mathrm{a}}$} \\
\hline & Non-Rel & SFR & SO1 & $\mathrm{SFR}+\mathrm{SO} 1$ & Full-Rel $^{\mathrm{b}}$ & & In $\mathrm{C}_{6} \mathrm{H}_{12}$ & Gas phase \\
\hline \multicolumn{9}{|c|}{${ }^{3} \Pi_{1 u}$ state $\left(n-\sigma^{*}\right)$} \\
\hline energy $^{c}$ & 16747 & 15533 & 15003 & 13562 & 13305 & 14312 & 13800 & \\
\hline$A^{\mathrm{d}}$ & 0.0 & 0.0 & -0.00004 & -0.0078 & -0.0085 & & -0.0320 & \\
\hline$B^{\mathrm{e}}$ & 0.0 & 0.0 & 0.0220 & 0.4759 & 0.6613 & & 0.183 & \\
\hline$D^{\mathrm{f}}$ & 0.0 & 0.0 & 0.0000 .73 & 0.0150 & 0.0162 & & 0.0639 & \\
\hline$f$ & 0.0 & 0.0 & $10^{-6}$ & 0.000095 & 0.00010 & & 0.00041 & \\
\hline \multicolumn{9}{|c|}{${ }^{3} \Pi_{0 u}$ state $\left(n-\sigma^{*}\right)$} \\
\hline energy & 16747 & 15533 & 14251 & 13056 & 12918 & 17457 & 18600 & 18600 \\
\hline$A$ & 0.0 & 0.0 & 0.0 & 0.0 & 0.0 & & 0.0 & 0.0 \\
\hline$B$ & 0.0 & 0.0 & -0.0401 & -0.4532 & -0.6393 & & -0.358 & -0.198 \\
\hline$D$ & 0.0 & 0.0 & 1.6677 & 1.8954 & 2.1495 & & 1.29 & 0.99 \\
\hline$f$ & 0.0 & 0.0 & 0.0112 & 0.0116 & 0.0131 & & 0.0113 & 0.0087 \\
\hline \multicolumn{9}{|c|}{${ }^{1} \Pi_{u}$ state $\left(n-\sigma^{*}\right)$} \\
\hline energy & 23751 & 22288 & 24684 & 23302 & 23470 & 20543 & 19700 & 19700 \\
\hline$A$ & $-10^{-6}$ & -0.0340 & -0.00007 & -0.0241 & -0.0233 & & -0.113 & -0.093 \\
\hline$B$ & -0.0004 & 0.1126 & -0.0024 & 0.0460 & 0.0418 & & 0.325 & 0.175 \\
\hline$D$ & $10^{-6}$ & 0.0680 & 0.00013 & 0.0460 & 0.0445 & & 0.226 & 0.186 \\
\hline$f$ & $<10^{-6}$ & 0.00071 & $10^{-6}$ & 0.00050 & 0.00049 & & 0.0021 & 0.0017 \\
\hline
\end{tabular}

${ }^{\mathrm{a}}$ Reference 27.

${ }^{\mathrm{b}} \mathrm{SFR}+\mathrm{SO} 1+\mathrm{SO} 2$ calculation.

${ }^{\mathrm{c}}$ Unit in $\mathrm{cm}^{-1}$.

${ }^{\mathrm{d}}$ Unit in $\mathrm{D}^{2} \mu \mathrm{B}$

${ }^{\mathrm{e}}$ Unit in $10^{-3} \mathrm{D}^{2} \mu \mathrm{B} / \mathrm{cm}^{-1}$.

${ }^{\mathrm{f}}$ Unit in $\mathrm{D}^{2}$.

TABLE V. SECI excitation energies, Faraday $A$ terms $(A)$, Faraday $B$ terms $(B)$, dipole strengths $(D)$, and oscillator strengths $(f)$ of $\mathrm{Br}_{2}$.

\begin{tabular}{|c|c|c|c|c|c|c|}
\hline & \multicolumn{3}{|c|}{ GUHF-SECI } & \multirow{2}{*}{$\begin{array}{c}\text { SAC-Cl } \\
\text { SO1 }\end{array}$} & \multicolumn{2}{|c|}{ Expt. $^{\text {a }}$} \\
\hline & Non-Rel & $\mathrm{SFR}+\mathrm{SO} 1$ & Full-Rel $^{\mathrm{b}}$ & & In $\mathrm{CCl}_{4}$ & Gas phase \\
\hline \multicolumn{7}{|c|}{${ }^{3} \Pi_{1 u}$ state $\left(n-\sigma^{*}\right)$} \\
\hline energy ${ }^{c}$ & 20266 & 18785 & 18880 & 18515 & 18500 & 18500 \\
\hline$A^{\mathrm{d}}$ & 0.0 & -0.0031 & -0.0026 & & -0.0172 & -0.0127 \\
\hline$B^{\mathrm{e}}$ & 0.0 & 0.0740 & 0.0644 & & 0.059 & 0.081 \\
\hline$D^{\mathrm{f}}$ & 0.0 & 0.0062 & 0.0051 & & 0.0344 & 0.0254 \\
\hline$f$ & 0.0 & 0.000054 & 0.000046 & & 0.00030 & 0.00020 \\
\hline \multicolumn{7}{|c|}{${ }^{3} \Pi_{0 u}$ state $\left(n-\sigma^{*}\right)$} \\
\hline energy & 20266 & 17774 & 17915 & 20015 & 20800 & 20800 \\
\hline$A$ & 0.0 & 0.0 & 0.0 & & 0.0 & 0.0 \\
\hline$B$ & 0.0 & -0.0637 & -0.0557 & & -0.079 & -0.069 \\
\hline$D$ & 0.0 & 0.2652 & 0.2168 & & 0.084 & 0.072 \\
\hline$f$ & 0.0 & 0.0022 & 0.0018 & & 0.00082 & 0.00070 \\
\hline \multicolumn{7}{|c|}{${ }^{1} \Pi_{u}$ state $\left(n-\sigma^{*}\right)$} \\
\hline energy & 28313 & 27263 & 27221 & 24570 & 24100 & 24100 \\
\hline$A$ & -0.0165 & -0.0501 & -0.0507 & & -0.165 & -0.119 \\
\hline$B$ & 0.0443 & 0.0665 & 0.0687 & & 0.101 & 0.076 \\
\hline$D$ & 0.0330 & 0.0985 & 0.1001 & & 0.331 & 0.237 \\
\hline$f$ & 0.00044 & 0.0013 & 0.0013 & & 0.0038 & 0.0027 \\
\hline
\end{tabular}

${ }^{\mathrm{a}}$ Reference 27.

${ }^{\mathrm{b}} \mathrm{SFR}+\mathrm{SO} 1+\mathrm{SO} 2$ calculation.

${ }^{\mathrm{c}}$ Unit in $\mathrm{cm}^{-1}$.

${ }^{\mathrm{d}}$ Unit in $\mathrm{D}^{2} \mu \mathrm{B}$.

${ }^{\mathrm{e}}$ Unit in $10^{-3} \mathrm{D}^{2} \mu \mathrm{B} / \mathrm{cm}^{-1}$.

${ }^{\mathrm{f}}$ Unit in $\mathrm{D}^{2}$. 
TABLE VI. SECI excitation energies, Faraday $A$ terms $(A)$, Faraday $B$ terms $(B)$, dipole strengths $(D)$, and oscillator strengths $(f)$ of $\mathrm{Cl}_{2}$.

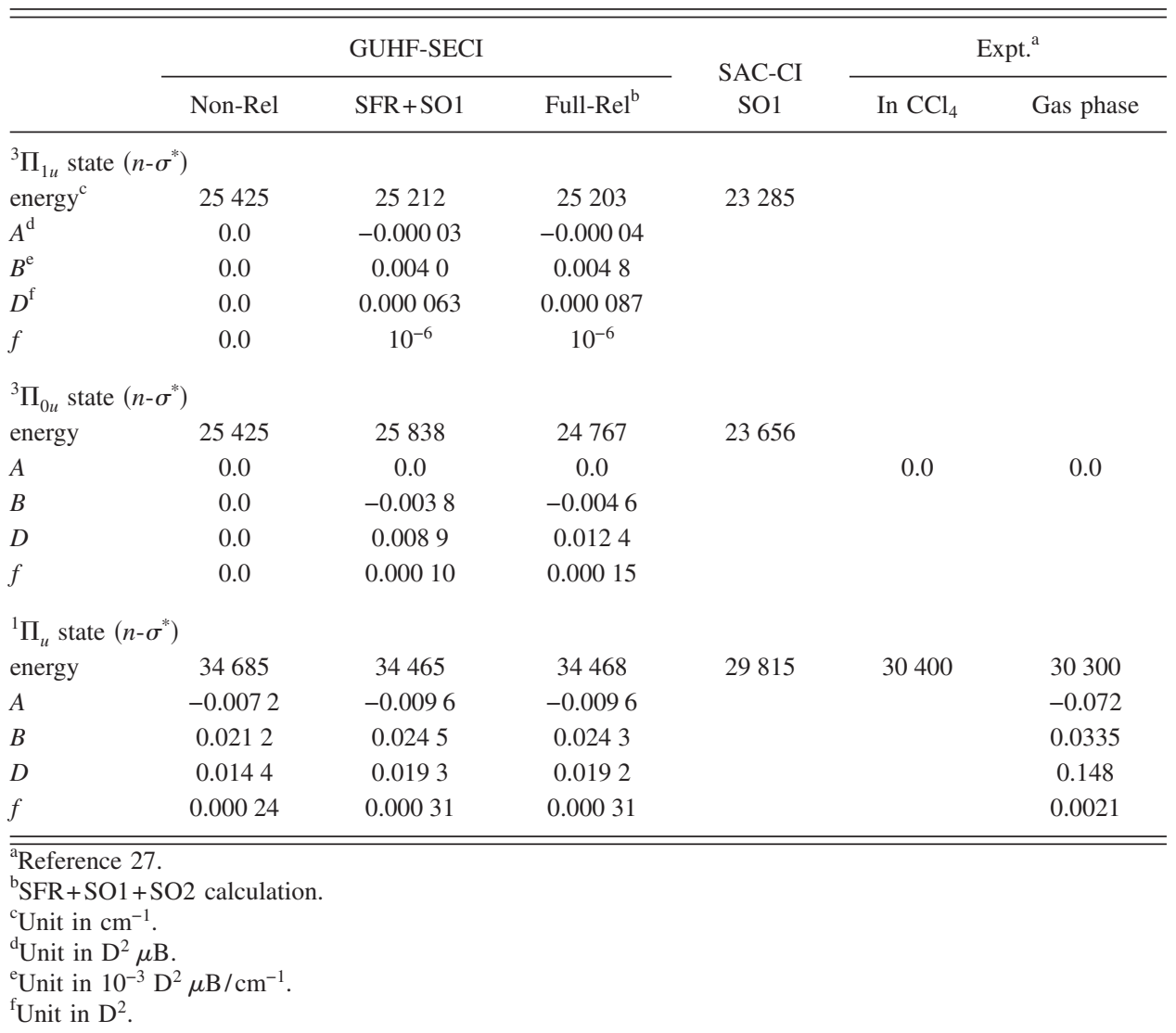

in the nonrelativistic limit and gain transition intensity through SO interactions with singlet states. The lowest band for $\mathrm{Cl}_{2}$ is, on the other hand, thought to be truly one band (or the SO interaction with the two triplet states is small) and is assigned to ${ }^{1} \Pi_{u}$. Although the experimental results for $\mathrm{F}_{2}$ have not been reported, the SO interaction for $F_{2}$ is also expected to be very small.

The SECI excitation energies, MCD Faraday $A$ and $B$ terms, dipole strengths, and oscillator strengths of the three excited states, ${ }^{3} \Pi_{1 u},{ }^{3} \Pi_{0 u}$, and ${ }^{1} \Pi_{u}$, in all four halogen molecules are shown in Tables IV-VII. In Figs. 1 and 2, the calculated Faraday $B$ and $A$ terms at the various relativistic levels are plotted with their corresponding experimental values. These terms were calculated by the Non-Rel SFR +SO1, and Full-Rel GUHF/SECI methods. In addition, SFR and SO1 calculations were also performed for $\mathrm{I}_{2}$. The experimental values ${ }^{27}$ were determined by a curve-fitting procedure. Since the ground states are nondegenerate, the Faraday $C$ terms vanished for all the molecules.

For $\mathrm{I}_{2}$ (in Table IV), the $B$ term of the ${ }^{1} \Pi_{u}$ state was significantly improved from the Non-Rel value of $-0.0004 \mathrm{D}^{2} \mu \mathrm{B} / \mathrm{kK}$ to the Full-Rel value of 0.0418 , which approaches the experimental values of 0.325 and 0.175 . The $A$ term was also improved from $-10^{-6} \mathrm{D}^{2} \mu \mathrm{B}$ in Non-Rel to -0.0233 in Full-Rel, and this is mainly due to the change in the dipole strength of ${ }^{1} \Pi_{u}$ from $10^{-6} \mathrm{D}^{2}$ in Non-Rel to 0.0445 in Full-Rel. Furthermore, the Faraday terms of the ${ }^{3} \Pi_{1 u}$ and ${ }^{3} \Pi_{0 u}$ states appear only in the relativistic calculations, because these are derived from the triplet excitations.
The signs of the calculated $A$ and $B$ terms were in accord with the experimental data. When the SO1 terms are considered, the Faraday terms of ${ }^{3} \Pi_{1 u}$ and ${ }^{3} \Pi_{0 u}$ appeared, but the values in the SO1 calculation were quite different from the Full-Rel values. In addition, even the signs of the values in the SO1 calculation were different from those in Full-Rel for the $B$ term of ${ }^{1} \Pi_{u}$. On the other hand, for ${ }^{1} \Pi_{u}$, the SFR calculation gave the values of the Faraday terms that are similar to those obtained using Full-Rel. This implies that the SFR terms improve the calculations of the Faraday terms for all states, while the SO1 terms are essential for the calculations involving the triplet-dominant states $\left({ }^{3} \Pi_{1 u}\right.$ and $\left.{ }^{3} \Pi_{0 u}\right)$. The Faraday terms are not additive with respect to the SFR and SO1 terms, and so their simultaneous consideration is important. The SFR+SO1 calculation gave 0.0460 for the $B$ term of the ${ }^{1} \Pi_{u}$ state, which is comparable with the Full-Rel value of 0.0418 . Also for the ${ }^{3} \Pi_{1 u}$ and ${ }^{3} \Pi_{0 u}$ states, the $\mathrm{SFR}+\mathrm{SO} 1$ calculations improved the $B$ term from the NonRel values of 0.0220 and -0.0401 to 0.4759 and -0.4532 , respectively. These SFR+SO1 values had the same order as the Full-Rel values, namely, 0.6613 and -0.6393 . In contrast, the differences between the SFR+SO1 and Full-Rel results were smaller than the difference between the Non-Rel and SFR+SO1 results, although these former differences were not negligible. This is consistent with our previous results for $\mathrm{CH}_{3} \mathrm{I}^{34}$

For $\mathrm{Br}_{2}$ in Table $\mathrm{V}$, the relativistic effects also influenced the Faraday $A$ and $B$ terms. For ${ }^{1} \Pi_{u}$, the $B$ term changed from the Non-Rel value of 0.0443 to the SFR+SO1 value of 
TABLE VII. SECI excitation energies, Faraday $A$ terms $(A)$, Faraday $B$ terms $(B)$, dipole strengths $(D)$, and oscillator strengths $(f)$ of $\mathrm{F}_{2}$.

\begin{tabular}{|c|c|c|c|c|}
\hline & \multicolumn{3}{|c|}{ GUHF-SECI } & \multirow{2}{*}{$\begin{array}{c}\text { SAC-Cl } \\
\text { SO1 }\end{array}$} \\
\hline & Non-Rel & $\mathrm{SFR}+\mathrm{SO} 1$ & Full-Rel $^{\mathrm{a}}$ & \\
\hline \multicolumn{5}{|c|}{${ }^{3} \Pi_{1 u}$ state $\left(n-\sigma^{*}\right)$} \\
\hline energy ${ }^{b}$ & 25919 & 25823 & 25813 & 24511 \\
\hline$A^{\mathrm{c}}$ & 0.0 & $>-10^{-6 \mathrm{~d}}$ & $>-10^{-6 \mathrm{~d}}$ & \\
\hline$B^{\mathrm{e}}$ & 0.0 & 0.00003 & 0.00002 & \\
\hline$D^{\mathrm{f}}$ & 0.0 & $<10^{-6}$ & $<10^{-6}$ & \\
\hline$f$ & 0.0 & $<10^{-6}$ & $<10^{-6}$ & \\
\hline \multicolumn{5}{|c|}{${ }^{3} \Pi_{0 u}$ state $\left(n-\sigma^{*}\right)$} \\
\hline energy & 25919 & 25600 & 25532 & 24715 \\
\hline$A$ & 0.0 & 0.0 & 0.0 & \\
\hline$B$ & 0.0 & -0.00004 & -0.00005 & \\
\hline$D$ & 0.0 & 0.0012 & 0.0022 & \\
\hline$f$ & 0.0 & 0.000015 & 0.000026 & \\
\hline \multicolumn{5}{|c|}{${ }^{1} \Pi_{u}$ state $\left(n-\sigma^{*}\right)$} \\
\hline energy & 40020 & 39986 & 40031 & 34876 \\
\hline$A$ & -0.0005 & -0.0003 & -0.0003 & \\
\hline$B$ & -0.0047 & -0.0040 & -0.0039 & \\
\hline$D$ & 0.00091 & 0.00066 & 0.00064 & \\
\hline$f$ & 0.000017 & 0.000012 & 0.000012 & \\
\hline
\end{tabular}

${ }^{\mathrm{S} F R}+\mathrm{SO} 1+\mathrm{SO} 2$ calculation.

${ }^{\mathrm{b}}$ Unit in $\mathrm{cm}^{-1}$.

${ }^{c}$ Unit in $\mathrm{D}^{2} \mu \mathrm{B}$.

${ }^{\mathrm{d}}$ A negative value with the absolute value less than $10^{-6}$.

${ }^{\mathrm{e}}$ Unit in $10^{-3} \mathrm{D}^{2} \mu \mathrm{B} / \mathrm{cm}^{-1}$.

${ }^{\mathrm{f}}$ Unit in $\mathrm{D}^{2}$.

0.0665 , and lastly, the Full-Rel value of 0.0687 . The $A$ term was -0.0165 in Non-Rel, becoming -0.0501 and -0.0507 in SFR + SO1 and Full-Rel, respectively. For the ${ }^{3} \Pi_{1 u}$ and ${ }^{3} \Pi_{0 u}$ states, the $B$ terms in the relativistic calculations had positive and negative values, respectively, in accordance with the experimental results. The $\mathrm{SO} 2$ effect on the Faraday terms was smaller than the SFR+SO1 effect, similar to $\mathrm{I}_{2}$. As a whole, although the relativistic effects in $\mathrm{MCD}$ of $\mathrm{Br}_{2}$ were considerable, the shifts of the Faraday terms due to the relativistic treatment were smaller than for $\mathrm{I}_{2}$.

Tables VI and VII show the results for $\mathrm{Cl}_{2}$ and $\mathrm{F}_{2}$, respectively. For $\mathrm{Cl}_{2}$, the calculated values became closer to

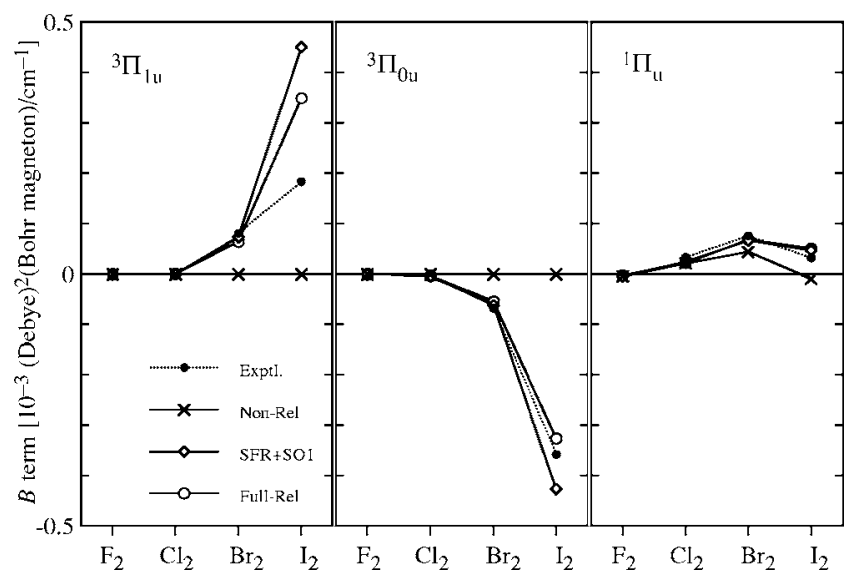

FIG. 1. Faraday $B$ terms of halogen molecules calculated by the GUHF/ SECI method compared with the experimental values.

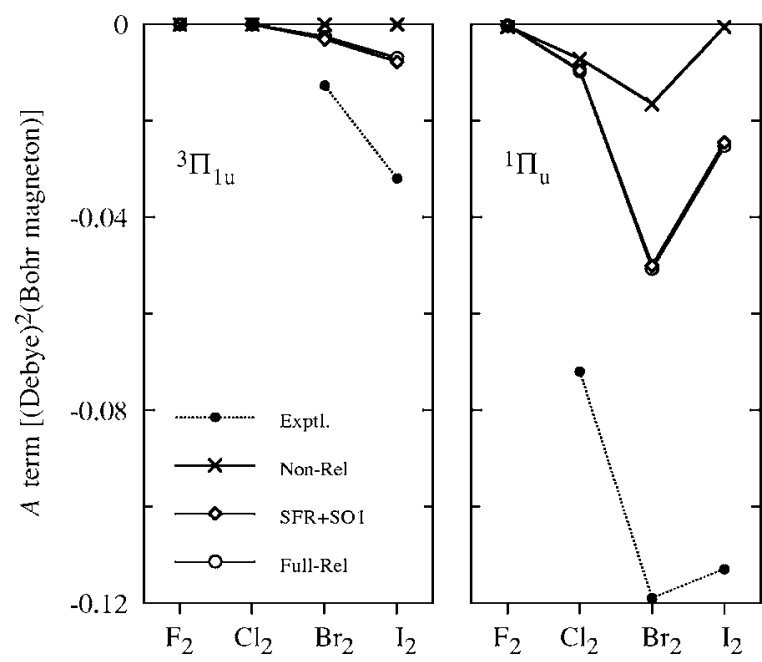

FIG. 2. Faraday $A$ terms of halogen molecules calculated by the GUHF/ SECI method compared with the experimental values.

the experimental results when the relativistic effects were considered. The $B$ term was improved from the Non-Rel value of 0.0212 to the Full-Rel value of 0.0243; the experimental value is 0.0335 . The $A$ term was similarly improved by the relativistic corrections. However, the changes were much smaller than for $\mathrm{I}_{2}$ or $\mathrm{Br}_{2}$. For $\mathrm{F}_{2}$, the Faraday terms for ${ }^{1} \Pi_{u}$ are almost unchanged by the relativistic effects, and those for ${ }^{3} \Pi_{1 u}$ and ${ }^{3} \Pi_{0 u}$ were almost zero even in the FullRel calculations. We thus conclude that the relativistic effects on MCD are important only for molecules including heavy atoms.

Although all the signs of the Faraday terms for the halogen molecules were correctly reproduced in the relativistic calculations, some absolute values of the calculated Faraday terms were quite different from the experimental values. This is mainly due to the poor description of the dipole strengths in these systems. These over/underestimations can be attributed to electron correlations. Furthermore, the order of the calculated energies is reversed from that of the experimental energies known for $\mathrm{I}_{2}$ and $\mathrm{Br}_{2}$; the assignments of ${ }^{3} \Pi_{1 u}$ and ${ }^{3} \Pi_{0 u}$ states in the GUHF/SECI calculations were made based on oscillator strengths rather than excitation energies. We performed SAC-CI calculations with the SO1 perturbation in order to confirm the validity of the assignments made by SECI and to obtain more accurate excitation energies, and these results are listed in Tables IV-VII. The SAC-CI + SO1 calculation corrects the energy orders, and we confirmed that the assignment made by the SECI method was correct, by configuration analysis. The energies calculated by SAC-CI+SO1 approached the corresponding experimental values. For $\mathrm{I}_{2}$, the excitation energies of the ${ }^{3} \Pi_{1 u},{ }^{3} \Pi_{0 u}$, and ${ }^{1} \Pi_{u}$ states were calculated to 14312,17457 , and $20543 \mathrm{~cm}^{-1}$, respectively, which are comparable to the experimental values: 13800,18600 , and $19700 \mathrm{~cm}^{-1}$. For $\mathrm{Br}_{2}$, the SAC-CI+SO1 energies were 18515,20015 , and $24570 \mathrm{~cm}^{-1}$, respectively, while the experimental values were 18 500, 20800 , and $24100 \mathrm{~cm}^{-1}$. The SAC-CI+SO1 calculations for $\mathrm{CH}_{3} \mathrm{I}$ also resulted in the same trend. ${ }^{34} \mathrm{Al}-$ though no experimental data are available for the ${ }^{3} \Pi_{1 u}$ and ${ }^{3} \Pi_{0 u}$ states of $\mathrm{Cl}_{2}$ and $\mathrm{F}_{2}$, the reverse order of their energies 
was observed in our calculations. Since we did not obtain accurate energies, we did not show theoretical spectra nor judged the validity of the experimental fitting procedures. Correlation effects and, in particular, the coupling between electron correlation and relativistic effects in MCD are interesting subjects for the study in the near future.

\section{CONCLUSIONS}

The generalized UHF (GUHF)/SECI method for calculating the Faraday terms in MCD with finite magnetic-field perturbation was presented and the results for ethylene, $p$-BQ, and $o$ - $\mathrm{BQ}$ were compared with those obtained by the conventional SOS method. The differences were not negligible, and even the signs of the terms obtained by the two methods were opposite for the lowest $\pi-\pi^{*}$ states of ethylene. Quasirelativistic treatments in the calculations of the Faraday terms were also discussed. The method was applied to the three $n-\sigma^{*}$ states $\left({ }^{3} \Pi_{1 u},{ }^{3} \Pi_{0 u}\right.$, and $\left.{ }^{1} \Pi_{u}\right)$ of each of the halogen molecules $\mathrm{I}_{2}, \mathrm{Br}_{2}, \mathrm{Cl}_{2}$, and $\mathrm{F}_{2}$. The relativistic effects became important for the MCD spectra of heavy molecules: the Faraday terms for $\mathrm{I}_{2}$ and $\mathrm{Br}_{2}$ were significantly changed by the relativistic corrections, whereas these effects were negligible for $\mathrm{Cl}_{2}$ and $\mathrm{F}_{2}$. The spin-free relativistic Hamiltonian incorporating the one-electron spin-orbit term gave the results similar to the full relativistic case, indicating that the relativistic effects induced by the two-electron spinorbit term in MCD are small. In all calculations, the signs of the Faraday terms agreed with those of the experimental values, although some of their absolute values were over/ underestimated compared to the experiments. This was thought to be mainly due to a lack of electron correlation.

\section{ACKNOWLEDGMENT}

Part of this study has been supported by a Grant-in-Aid for Scientific Research from the Ministry of Education Science Sports and Culture of Japan.

\footnotetext{
${ }^{1}$ A. Gedanken and O. Schnepp, Chem. Phys. Lett. 37, 373 (1976).

${ }^{2}$ M. Brith-Lindner and S. D. Allen, Chem. Phys. Lett. 47, 32 (1977)

${ }^{3}$ P. A. Snyder, P. N. Schatz, and E. M. Rowe, Chem. Phys. Lett. 110, 508 (1984)

${ }^{4}$ P. A. Snyder, R. W. C. Hansen, and E. M. Rowe, J. Phys. Chem. 100, 17756 (1996)

${ }^{5}$ A. Gedanken and O. Schnepp, Chem. Phys. 12, 341 (1976).

${ }^{6}$ I. N. Douglas, R. Grinter, and A. J. Thomson, Mol. Phys. 26, 1257 (1973).

${ }^{7}$ S. D. Allen, M. G. Mason, O. Schnepp, and P. J. Stephens, Chem. Phys. Lett. 30, 140 (1975).

${ }^{8}$ D. J. Shieh, S. H. Lin, and H. Eyring, J. Phys. Chem. 77, 1031 (1973).

${ }^{9}$ D. W. Miles and H. Eyring, Proc. Natl. Acad. Sci. U.S.A. 70, 3754 (1973).

${ }^{10}$ A. Kaito, A. Tajiri, and M. Hatano, Chem. Phys. Lett. 25, 548 (1974).

${ }^{11}$ A. Kaito, A. Tajiri, and M. Hatano, Chem. Phys. Lett. 28, 197 (1974).

${ }^{12}$ Y. H. Yoon, S. T. Lee, D. J. Shieh, H. Eyring, and S. H. Lin, Chem. Phys. Lett. 38, 24 (1976).

${ }^{13}$ A. R. Meier and G. H. Wagnière, Chem. Phys. 113, 287 (1987).

${ }^{14}$ R. A. Goldbeck, B.-R. Tolf, A. G. H. Wee, A. Y. L. Shu, R. Records, E.
}

Bunnenberg, and C. Djerassi, J. Am. Chem. Soc. 108, 6449 (1986).

${ }^{15}$ J. Waluk, M. Müller, P. Swiderek, M. Köcher, E. Vogel, G. Hohlneicher, and J. Michl, J. Am. Chem. Soc. 113, 5511 (1991).

${ }^{16}$ P. J. Stephens, R. L. Nowery, and P. N. Schatz, J. Chem. Phys. 55, 224 (1971).

${ }^{17}$ P. J. Stephens, J. Chem. Phys. 52, 3489 (1970).

${ }^{18}$ D. J. Shieh, S. H. Lin, and H. Eyring, J. Phys. Chem. 76, 1844 (1972).

${ }^{19}$ S. Coriani, C. Hättig, P. Jørgensen, and T. Helgaker, J. Chem. Phys. 113, 3561 (2000).

${ }^{20}$ S. Coriani, P. Jørgensen, A. Rizzo, K. Ruud, and J. Olsen, Chem. Phys. Lett. 300, 61 (1999).

${ }^{21}$ E. Goldstein, S. Vijaya, and G. A. Segal, J. Am. Chem. Soc. 102, 6198 (1980).

${ }^{22}$ G. Marconi, Chem. Phys. Lett. 146, 259 (1988).

${ }^{23}$ L. Seamans and J. Linderberg, Mol. Phys. 24, 1393 (1972).

${ }^{24} \mathrm{~J}$. Michl, Tetrahedron 40, 3845 (1984). Many references are referred to in this review article.

${ }^{25}$ See, e.g., J. Fleischhauer and J. Michl, J. Phys. Chem. A 104, 7776 (2000).

${ }^{26}$ H. Nakatsuji, J. Chem. Phys. 61, 3728 (1974).

${ }^{27}$ M. Brith, M. D. Rowe, O. Schnepp, and P. J. Stephens, Chem. Phys. 9, 57 (1975).

${ }^{28}$ A. Gedanken and M. D. Rowe, Chem. Phys. Lett. 34, 39 (1975).

${ }^{29}$ B. R. Johnson and J. L. Kinsey, J. Phys. Chem. 100, 18937 (1996).

${ }^{30}$ M. D. Rowe and A. Gedanken, Chem. Phys. 10, 1 (1975).

${ }^{31}$ P. Hafner and W. H. E. Schwarz, Chem. Phys. Lett. 65, 537 (1979).

${ }^{32}$ J. Wan, R. Fukuda, M. Hada, and H. Nakatsuji, J. Phys. Chem. A 105, 128 (2001).

${ }^{33}$ R. Fukuda, M. Hada, and H. Nakatsuji, Rec. Adv. Comp. Chem. (in press).

${ }^{34}$ Y. Honda, M. Hada, M. Ehara, H. Nakatsuji, J. Downing, and J. Michl, Chem. Phys. Lett. 355, 219 (2002).

${ }^{35}$ J. L. Duncan, I. J. Wright, and D. van Leberghe, J. Mol. Spectrosc. 42, 463 (1972).

${ }^{36}$ K. Hagen and K. Hedberg, J. Chem. Phys. 59, 158 (1973).

${ }^{37}$ A. L. Macdonald and J. Trotter, J. Chem. Soc., Perkin Trans. 2 4, 476 (1973)

${ }^{38}$ O. Kennard, H. M. Powell, D. H. Whiffen, A. A. McConnell, and G. M. Lander, in Tables of Interatomic Distances and Configuration in Molecules and Ions, edited by L. E. Sutton (The Chemistry Society, London, 1965), Special Publication No. 18.

${ }^{39}$ T. H. Dunning, Jr., J. Chem. Phys. 53, 2823 (1970).

${ }^{40}$ S. Huzinaga, J. Andzelm, M. Klobukowski, E. Radzio-Andzelm, Y. Sakai, and H. Tatewaki, Gaussian Basis Set for Molecular Calculations (Elsevier, Amsterdam, 1984).

${ }^{41}$ M. Jungen, J. Chem. Phys. 74, 750 (1981).

${ }^{42}$ A. D. McLean and G. S. Chandler, J. Chem. Phys. 72, 5639 (1980).

${ }^{43}$ S. Huzinaga and M. Klobukowski, Chem. Phys. Lett. 212, 260 (1993).

${ }^{44}$ B. A. Hess, Phys. Rev. A 33, 3742 (1986).

${ }^{45}$ R. Fukuda, M. Hada, and H. Nakatsuji, J. Chem. Phys. 118, 1015 (2003).

${ }^{46}$ R. Fukuda, M. Hada, and H. Nakatsuji, J. Chem. Phys. 118, 1027 (2003).

${ }^{47}$ K. Andersson, P.-Å. Malmqvist, B. O. Roos, A. J. Sadlej, and K. Wolinski, J. Phys. Chem. 94, 5483 (1990).

${ }^{48}$ K. Andersson, P.- $\AA$. Malmqvist, and B. O. Roos, J. Chem. Phys. 96, 1218 (1992)

${ }^{49}$ K. Andersson and B. O. Roos, in Advanced Series in Physical Chemistry: Modern Electron Structure Theory, edited by R. Yarkony (World Scientific, Singapore, 1995), Vol. 2, Pt. I, p. 55.

${ }^{50}$ H. Nakatsuji, Chem. Phys. Lett. 59, 362 (1978).

${ }^{51}$ H. Nakatsuji, Chem. Phys. Lett. 67, 329 (1979).

${ }^{52} \mathrm{H}$. Nakatsuji, in Computational Chemistry, Reviews of Current Trends, edited by J. Leszczynski (World Scientific, Singapore, 1997), Vol. 2.

${ }^{53}$ S. Fukawa, M. Hada, R. Fukuda, S. Tanaka, and H. Nakatsuji, J. Comput. Chem. 22, 528 (2001).

${ }^{54}$ P. A. Snyder, S. Atanasova, and R. W. C. Hansen, J. Phys. Chem. A 108, 4194 (2004). 